\title{
The relationship between the expression of PLIN3 and PLIN5 protein flowing endurance training in streptozotocin rats
}

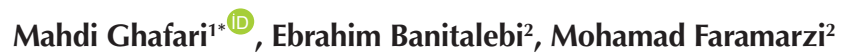 \\ ${ }^{1}$ Assistant Professor in Exercise Physiology, Shahrekord University, Shahrekord, Iran \\ ${ }^{2}$ Associate Professor in Exercise Physiology, Shahrekord University, Shahrekord, Iran
}

*Corresponding Author: Mahdi Ghafari, Assistant Professor in Exercise Physiology, Shahrekord University, Shahrekord, Iran. Tel: 038-32324401, Email: ghafari.mehdi@gmail.com, ghafari.mehdi@sku.ac.ir,

\begin{abstract}
Background and aims: Intermuscular lipolysis disorder plays an important role in insulin resistance and diabetes mellitus and perilipin PLIN5 and PLIN3 are the key proteins in regulating muscle cellular lipolysis. Therefore, the purpose of this study was to examine the relationship between the expression of PLIN3 and PLIN5 protein following endurance training in streptozotocin (STZ) rats.

Methods: A number of 24 male Wistar rats were randomly divided into low endurance training group $(n=8)$, high-intensity training group $(n=8)$, and control group $(n=8)$. Diabetes was induced in every rat by STZ injection. Three days after injection, the blood samples were taken from the cut tip of the tails of the mice and animals with blood glucose greater than $300 \mathrm{mg} / \mathrm{dL}$ were considered diabetic. The training program included eight weeks of aerobic training at different intensities. Training in high- and low-intensity groups included 22-25 and 5-8 $\mathrm{m} / \mathrm{min}$ of training. Finally, one-way analysis of variance (ANOVA) and correlation was used to determine the significance of the differences between variables, followed by utilizing Tukey's post-hoc test for significance.

Results: The comparison between the groups by ANOVA showed significant differences in PLIN3 $(P=0.0006)$ and PLIN5 $(P=0.012)$. The results of Tukey post hoc test also demonstrated a statistical difference between the mean values of diabetic control group and high-intensity endurance group regarding PLIN3 $(P=0.01)$ and PLIN5 $(P=0.009)$, but no significant increase was observed in the lowintensity exercise group as compared to the control group (PLIN3, $P=0.067 \&$ PLIN5, $P=0.44$ ). As regards insulin resistance, there was a significant difference among the three groups $(P=0.0001)$. Eventually, the result of the correlation between PLIN3 and PLIN5 showed similar enhancement by increasing the intensity $(P=0.0026)$.

Conclusion: According to research results, high-intensity endurance training increased the expression of PLIN3 and PLIN5 in diabetic specimens and PLIN3 and PLIN5 followed a similar increase pattern in high-intensity training.

Keywords: Diabetes, PLIN5, PLIN3, Endurance Training, Intramuscular Triglycerides, Insulin Resistance
\end{abstract}

Received: 31 July 2017, Accepted: 27 January 2018, ePublished: 30 August 2019

\section{Introduction}

Diabetes is characterized by excessive fat accumulation in the form of triglycerides in non-adipose tissues such as liver, skeletal muscle, and heart (1). In addition, intramuscular triglyceride (IMTG) is associated with insulin resistance in diabetic patients (2). The concept of "lipid-induced skeletal muscle insulin resistance" demonstrates a correlation between IMTG concentrations and insulin resistance (3), as well as the relationship between IMTG and insulin resistance in individuals with low-oxidative capacity and low mobility. The low levels of IMTG are observed by increased insulin resistance as well (4). Recent evidence has shown that cytosolic regulating lipid droplet (LD) proteins play crucial roles in important cellular processes such as cellular energy homeostasis lipid storage (5). The PLIN family including PLIN1 to PLIN5 is the best-characterized family of LD proteins (6). The results of some studies showed that the content of perilipins in type 1 muscle fibers is more than type 2 muscle fibers $(7,8)$. Similarly, the IMTG alternate in exercise conditions is independent of the changes in PLIN protein in diabetic patients who may represent the potential role of PLIN proteins in insulin resistance. According to previous studies, PLIN3 and PLIN5 are used in intensive training and moderate-intensity exercises $(8,9)$, while some studies indicated that the effect of training exercises on the amount of IMTG is unclear.

Although some studies reported an increase in the content of IMTG in obese patients with type 2 diabetes $(10,11)$, other studies failed to report these changes $(4,11)$. Furthermore, it is found that training with different intensities has different effects on the muscle LD $(12,13)$, while Shepherd et al demonstrated that high-intensity training improves IMTG in the same way (8). Different 
expressions of skeletal muscle PLIN protein are reported in the training as well.

PLIN3 and PLIN5 show engagement as the potential mediators of fatty acid or LD interaction with skeletal muscle mitochondria (14) since they are both exchangeable LD proteins that interact with intracellular organelle (14). Moreover, PLIN3 has a known role in vesicular moving in cell culture models (15) and PLIN5 is localized to mitochondria in skeletal muscle models. However, issues such as the nature of their association and what might occur under lipolytic conditions are unknown (14). PLIN5 is regarded as an LD protein, which is highly expressed in oxidative tissue $(16,17)$ and regulates lipid. Previous researches show that the size of LD is related to PLIN5 expression and the excess accumulation of intramuscular fat is associated with such conditions as insulin resistance and being afflicted with type 2 diabetes (18).

The intensity of physical activity is considered an important factor for diabetic patients (7). Evidence suggests that more IMTG is consumed and replenished in high-intensity interval training. Additionally, PLIN3 and PLIN5 proteins play a role in the hydrolysis of triglycerides stored in muscle LDs and the reduced turnover of IMTG is related to insulin resistance. On the other hand, considering that endurance training is able to increase IMTG, the current study sought to investigate the relationship between the expression of PLIN3 and PLIN5 proteins following endurance training in streptozotocin rats.

\section{Materials and Methods}

The current research is an experimental in vitro study. A total of 24 eight-week male Wistar rats were obtained from the Pasteur Institute Animal Care Center in Karaj, Iran. After transferring the mice to the laboratory, they were randomly divided into diabetic control, low-intensity, and high-intensity endurance training. Throughout the study, animals were maintained in normal conditions $(12 \mathrm{~L} / 12 \mathrm{D}$ at $23 \pm 3^{\circ} \mathrm{C}$ ) and fed with a standard laboratory diet, received enough water, and were housed under the same maintenance and laboratory conditions. After a few days, animals were weighed and then anesthetized with ether, and finally, received a single intraperitoneal injection of streptozotocin (STZ) at a dose of $55 \mathrm{mg} / \mathrm{kg}$ body weight. Further, $9.5 \mathrm{mg}$ citrate buffer with a $\mathrm{pH}$ of 4.5 (sterile) was added per $1 \mathrm{~g} \mathrm{STZ}$ to prepare the solution and then a yellow solution was obtained after dissolving. Prior to STZ injection, a drop of blood was taken from the cut tip of the tail of the animal in order to determine STZ injection dose. Using blood glucose (BG) test strip and BG meter, fasting blood sugar level was then measured, followed by performing the injection. Next, the blood drop was taken through the tail-tip amputation method and the blood sugar level of the animal was measured as well. Accordingly, those animals with BG greater than
$300 \mathrm{mg} / \mathrm{dL}$ were considered diabetic. After one week and getting familiar with the laboratory environment, rats were randomly assigned to two training groups and one control group. Then, the rats became familiar with how to run on a treadmill for one week at a speed of $3 \mathrm{~m} / \mathrm{min}$ for 15-20 minutes. The control group rats participated in no training, but in experimental groups, the training protocol was performed during eight weeks for four days (per week) for 30 minutes as follows.

- Low-intensity group. Eight rats received training at a speed of $5-8 \mathrm{~m} / \mathrm{min}$ equivalent to $50 \%-60 \%$ $\mathrm{Vo}_{2 \max }$.

- High-intensity group. A number of 8 rats received training at a speed of $22-25 \mathrm{~m} / \mathrm{min}$ equivalent to $80 \% \mathrm{Vo}_{2 \max }$.

After performing the training protocol, all the rats were weighed 48 hours after the last training session program and then the rats were anesthetized using the intraperitoneal injections of ketamine $(90 \mathrm{mg} / \mathrm{kg})$ and xylazine $(10 \mathrm{mg} /$ $\mathrm{kg}$ ). Next, the blood samples ( $5 \mathrm{cc}$ ) were directly extracted from the hearts of the mice and entered into sterile tubes, and after one hour of room temperature storage, serum isolation was conducted using centrifugation method (for 10 minutes at $2500 \mathrm{rpm}$ ), and the isolated serum was frozen and kept in nitrogen at $-180^{\circ} \mathrm{C}$. In the post-test stage, all the collected blood samples were withdrawn in one day from the refrigerator and underwent the given tests based on the related protocol. Glucose levels were measured by Germany glucometer by cutting the tip of the tail. Further, the plasma levels of insulin were estimated by ELISA kit (rhizosphere, China with 5 microns per milliliter sensitivity and the coefficient of variation of $36 \%$ ). Finally, insulin resistance was calculated applying the homeostatic model assessment-insulin resistance (HOMA-IR) method by measuring insulin and fasting glucose according to the following formula (19).

HOMA-IR=fasting insulin $(\mathrm{ng} / \mathrm{mL}) \times$ fasting $B G(\mathrm{mg} /$ dL)/22.5

Similarly, to analyze the soleus muscle protein expression of perilipin 5 by western blot, approximately $50 \mathrm{mg}$ of each soleus skeletal muscle piece was powdered with a pestle in liquid nitrogen and lysed using a $1 \mathrm{~mL}$ of phosphate-buffered saline. Tissue homogenates were centrifuged at 12000 rpm for 15 minutes at $4^{\circ} \mathrm{C}$ and supernatant was removed as well. The total protein content of the tissue extract was determined by the Bradford method using bovine serum albumin. Accordingly, $50 \mu \mathrm{g}$ of protein was collected per sample, separated by SDS-PAGE in $8 \%$ polyacrylamide, and electrotransferred to polyvinylidene difluoride membranes. Next, the membranes were incubated in the blocking solution ( $5 \%$ milk) at room temperature for 2 hours. The membranes were then incubated with primary antibodies including PLIN3 (TIP47 Antibody (B-3) 200 
$\mu \mathrm{g} / \mathrm{mL}$, Santa Cruz) and PLIN5 (guinea pig polyclonal, Progen, \#GP31, Heidelberg, Germany), and then the peroxidase-conjugated secondary antibody was directed against the primary antibody. Ultimately, the membranes were developed by an enhanced chemiluminescence western blot detection system.

Data were reported as means \pm standard error (SE) values. To compare the groups, all dependent variables were analyzed by one-way ANOVA. Furthermore, Tukey post hoc test was used to determine significant differences among the groups. Data analysis was performed using SPSS software, version 21 and statistical significance was set at $P<0.05$.

\section{Results}

At first, there was no significant difference between the weighted averages (Table 1). No significant difference was observed between the weights in the control and training groups $(P=0.47)$ as well (Table 2$)$. However, the results of one-way ANOVA (Table 3) showed a significant difference in PLIN3 $(P=0.01)$, PLIN5 $(P=0.0122)$, glucose $(P=0.001)$, insulin $(P=0.001)$, and insulin resistance $(P=0.0001)$. As shown in Table 4 and Figure 1 , the results of Tukey post-hoc test also indicated that high-intensity endurance training had a significant effect on PLIN3 expression, $(P=0.01)$ while no significant increase in PLIN3 was observed in low-intensity training compared to control group $(P=0.67)$. Based on the results of Tukey post-hoc test, high-intensity endurance training demonstrated a significant impact on PLIN5 expression $(P=0.01)$. No significant increase in PLIN5 was observed in low-intensity training when compared to the control group $(P=0.44)$; the related details are presented in Table 5 and Figure 2. Table 6 shows the Tukey analysis of glucose, insulin serum levels, and insulin resistance. Table 7 and Figure 3 also represent the correlation between PLIN3 and PLIN5.

Table 1. Basic characteristics

\begin{tabular}{lccc}
\hline Values & Diabetic Control Group (n=8) & Low-intensity Diabetic Group (n=8) & High-intensity Diabetic Group $(\mathbf{n}=\mathbf{8})$ \\
\hline Weight before intervention $(\mathrm{g})$ & $201.10 \pm 14.700$ & $271.62 \pm 24.017$ & $182.90 \pm 17.026$ \\
Weight after intervention $(\mathrm{g})$ & $191.50 \pm 15.464$ & $186.50 \pm 42.578$ & $15.366 \pm 172.88$ \\
Glucose before interventions $(\mathrm{mg} / \mathrm{dL})$ & $450.68 \pm 25.38$ & $466.66 \pm 20.78$ & $431.6 \pm 25.3$ \\
\hline
\end{tabular}

Table 2. The results of covariance analysis, body mass changes after eight weeks of training with different intensities

\begin{tabular}{llccc}
\hline Variable & Group & $\begin{array}{c}\text { Diabetic Control Group } \\
(\mathbf{n = 8})\end{array}$ & $\begin{array}{c}\text { Low-Intensity Diabetic } \\
\text { Group (n=8) }\end{array}$ & $\begin{array}{c}\text { High-Intensity Diabetic } \\
\text { Group (n=8) }\end{array}$ \\
\hline \multirow{2}{*}{ Weight $(\mathrm{kg})$} & Pre-test & $201.10 \pm 14.700$ & $271.62 \pm 24.017$ & $\begin{array}{c}\boldsymbol{P} \text { Between } \\
\text { Groups }\end{array}$ \\
& Post-test & $191.50 \pm 15.464$ & $186.50 \pm 42.578$ & $172.88 \pm 17.026$ \\
& P-inside group & 0.75 & 0.51 & 0.15 .366 \\
\hline
\end{tabular}

Table 3. The Results of ANOVA analysis of PLIN3, PLIN5, Insulin, and HOHA-IR

\begin{tabular}{|c|c|c|c|c|c|}
\hline \multirow{3}{*}{ Variables } & \multicolumn{3}{|c|}{ Groups } & \multirow{2}{*}{\multicolumn{2}{|c|}{ ANOVA }} \\
\hline & $\begin{array}{l}\text { High-Intensity Diabetic Group } \\
\qquad(\mathrm{n}=8)\end{array}$ & $\begin{array}{l}\text { Low-Intensity Diabetic Group } \\
(\mathbf{n}=\mathbf{8})\end{array}$ & $\begin{array}{l}\text { Diabetic Control } \\
\text { Group }(n=8)\end{array}$ & & \\
\hline & Mean \pm SD & Mean \pm SD & Mean \pm SD & $\mathbf{F}$ & $\boldsymbol{P}$ \\
\hline PLIN3 (arbitrary) & $6196 \pm 2490$ & $4035 \pm 2402$ & $3400.56 \pm 2497.21$ & 5.54 & 0.0006 \\
\hline PLIN5 (arbitrary) & $7294.61 \pm 1283.85$ & $5292.22 \pm 2362.30$ & $3400.56 \pm 2497.21$ & 5.54 & $0.012^{*}$ \\
\hline Glucose (mg/dL) & $341.50 \pm 91.905$ & $0.1213 \pm 0.009$ & $557.75 \pm 158.847$ & 18.892 & $0.001^{*}$ \\
\hline Insulin & $0.1213 \pm 0.009$ & $0.1725 \pm 0.0310$ & $0.1925 \pm 0.036$ & 211.35 & $0.001^{*}$ \\
\hline HOMA-IR & $1.823 \pm 0.4415$ & $3.808 \pm 0.6883$ & $4.833 \pm 1.227$ & 25.84 & $0.0001^{*}$ \\
\hline
\end{tabular}

ANOVA: Analysis of variance; HOMA-IR: The homeostatic model assessment-insulin resistance; *Significant at $P \leq 0.05$.

Table 4. The results of Tukey post hoc test on the expression of PLIN3

\begin{tabular}{lcc}
\hline Group & Group & Different Mean \\
\hline High-intensity diabetic group $(\mathrm{n}=8)$ & Diabetic control group $(\mathrm{n}=8)$ & 992.5 \\
& Low-intensity diabetic group $(\mathrm{n}=8)$ & $0.01^{*}$ \\
Low-intensity diabetic group $(\mathrm{n}=8)$ & Diabetic control group $(\mathrm{n}=8)$ & 2157 \\
\hline
\end{tabular}

*Significant at $P \leq 0.05$. 
PLIN3

GAPDH
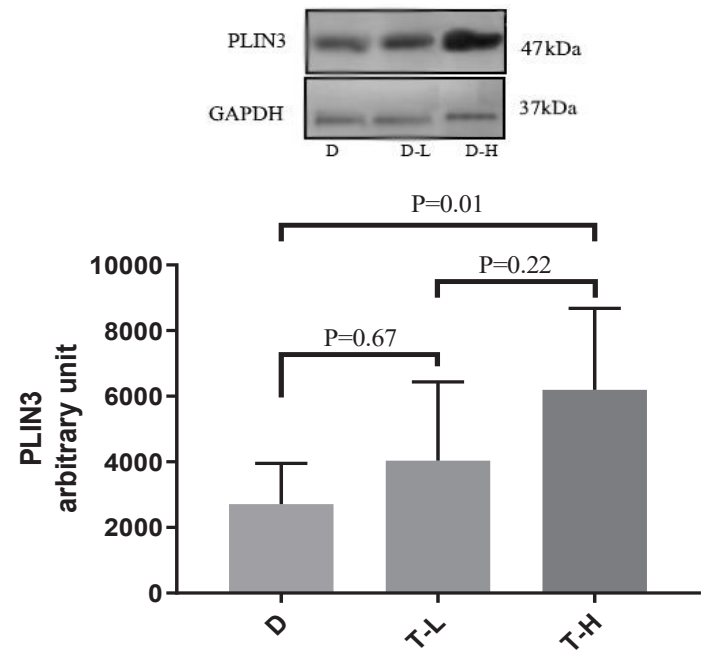

Figure 1. The effect of training on the expression of PLIN3 in soleus muscle. Note. D: Diabetic control; T-L: Low-intensity exercise; T-H: High-intensity exercise.

\section{Discussion}

Based on the results of this study, high-intensity exercise significantly increased the expression of PLIN3 and PLIN5 compared to the diabetic control group and PLIN protein increasing by high-intensity endurance training as well. STZ-induced diabetes causes muscle atrophy due to the increased levels of glucose and a decrease in insulin levels $(20,21)$. Moreover, muscle atrophy reduces protein synthesis and increases protein degradation in skeletal muscle (22). It seems that high-intensity training has a stimulatory effect to compensate for PLIN3 and PLIN5 protein synthesis (23). Minnaard et al also highlighted the benefits of training on PLIN5 protein for the muscle rate (24). The increased PLIN3 and PLIN5 expressions were reported in animal (14) and human (25) studies after training. It was reported that PLIN5 mitochondria increase after 30 minutes of high-intensity contraction, as well as fatty acid (FA) transport and metabolism in the muscle tissue (14). Similarly, Mason et al observed the accumulation of PLIN5 and PLIN3 protein content in Vastus lateralis muscle after 60 minutes of high-intensity training (26). Another study also showed an increase
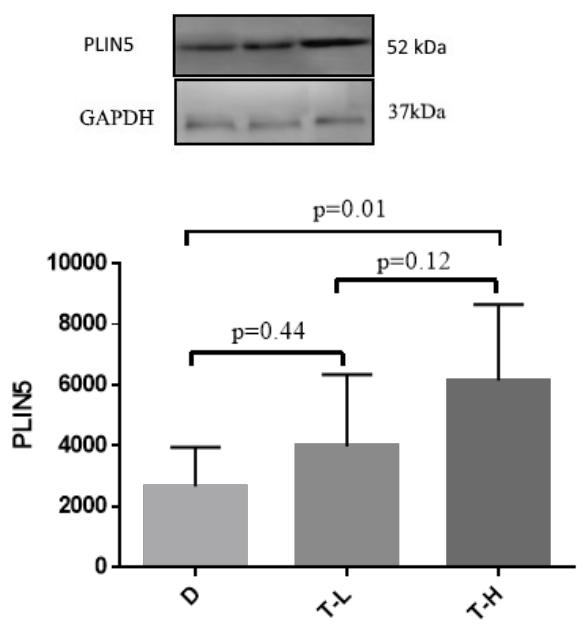

Figure 1. The effect of training on the expression of PLIN5 in soleus muscle. Note. D: Diabetic control; T-L: Low-intensity exercise; T-H: High-intensity exercise.

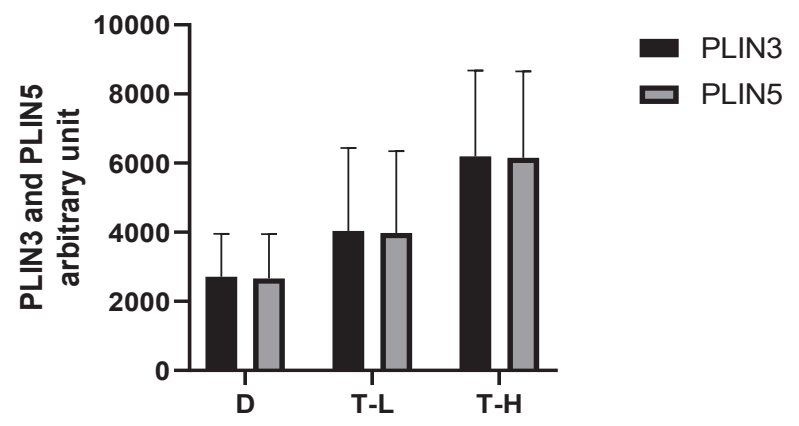

Figure 3. The relationship between PLIN3 and PLIN5 in different intensity. Note. D: Diabetic control; T-L: Low-intensity exercise; T-H: High-intensity exercise.

in PLIN5 in diabetic patients after 6 months of highendurance training (27).

Cell culture studies demonstrate that PLIN3 and PLIN5 expression recruits adipose triglyceride lipase (ATGL) and its coactivator, as the comparative gene identification-58 (CGI-58), to the LD surface under basal conditions. It is believed that PLIN5 phosphorylation releases CGI58 to bind ATGL to stimulate lipolysis in response to

Table 5. The results of Tukey post hoc test on the expression of PLIN5

\begin{tabular}{lcc}
\hline Group & Group & Different Mean \\
\hline High-intensity diabetic group $(\mathrm{n}=8)$ & Diabetic control group $(\mathrm{n}=8)$ & 3492.85 \\
& Low-intensity diabetic group $(\mathrm{n}=8)$ & $0.01^{*}$ \\
Low-intensity diabetic group $(\mathrm{n}=8)$ & Diabetic control group $(\mathrm{n}=8)$ & 2175.71 \\
\hline
\end{tabular}

*Significant at $P \leq 0.05$.

Table 6. The results of Tukey post hoc analysis of serum insulin and glucose levels

\begin{tabular}{llcccc}
\hline \multirow{2}{*}{ Group } & Group & \multicolumn{2}{c}{ Insulin } & \multicolumn{2}{c}{ Glucose } \\
\cline { 3 - 5 } & & Different Mean & Significant & Different Mean & Significant \\
\hline \multirow{2}{*}{ High-intensity diabetic group $(n=8)$} & Diabetic control group $(n=8)$ & 0.0712 & $0.000^{*}$ & 236.25 & $0.001^{*}$ \\
\cline { 3 - 6 } & Low-intensity diabetic group $(\mathrm{n}=8)$ & 0.0512 & $0.011^{*}$ & 155.5 & $0.046^{*}$ \\
\hline
\end{tabular}


Table 7. Correlation Between PLIN3 and PLIN5

\begin{tabular}{lccc}
\hline Pearson $r$ & PLIN5 T-H vs. PLIN3 T-H & PLIN5 T-L vs. PLIN3 T-L & Total PLIN3 vs. PLIN5 \\
\hline $\mathrm{R}$ & 0.99 & 0.99 & 1.000 \\
R-squared & 0.99 & 0.99 & 1.000 \\
$P$ value & 0.0001 & 0.0001 & 0.0026 \\
\hline
\end{tabular}

T-L: Low-intensity exercise; T-H: High-intensity exercise.

protein kinase A activation (18). Both PLIN3 and PLIN5 co-precipitated with CGI-58 at rest and following the contraction. MacPherson et al found that PLIN3 and PLIN5 proteins work together to regulate lipolysis (23). It seems PLIN5 regulates oxidative LD hydrolysis and controls the local FA flux to protect mitochondria against excessive exposure to FA during physiological stress, and therefore, reduces the insulin resistance (28).

The results of the present study revealed that low-intensity endurance training led to an increase in the expression of PLIN3 and PLIN5, but this increase was not significant while in the study by Mason et al, a significant increase was detected in endurance training with $60 \% \mathrm{Vo}_{2 \max }$ (27). In contrast, no significant increase was observed in PLIN3 and PLIN5 by low-intensity interval training, and it is assumed that a limited number of muscle fibers are used by this training due to less consumption of IMTG (27).

The comparison between endurance training exercises and high-intensity interval training showed that the latter leads to more accumulation and greater fragmentation of IMTG. It also increases the expression of PLIN3 and PLIN5 and probably maintains a low concentration of muscle FA metabolites, leading to improved insulin sensitivity in endurance training (28). Our findings further indicated that insulin resistance, along with low-intensity and highintensity endurance activities in diabetic rats, decreased significantly. Similarly, the destruction of pancreas by STZ leads to a sharp decrease in insulin levels, and owing to hyperglycemia (20), the loss of muscle mass is observed in the models of a severe decrease in insulin (30). Frequent impulses during training are also shown to coordinate the enzymes associated with the metabolism of IMTG (31), because of which endurance-trained athletes have high amounts of IMTG but are insulin sensitive. Further, the increased synthesis rates of IMTG were associated with decreased ceramide and diacylglycerol concentration (14). These data suggest that IMTG may protect against insulin resistance during increased free FA uptake (32).

\section{Conclusion}

In general, the obtained data demonstrated that the levels of PLIN3 and PLIN5 increase in response to high-intensity endurance training and thus decrease insulin resistance. Furthermore, the expression of the skeletal muscle PLIN5 protein relies on the intensity of training and both of these proteins have almost an ascending trend.
Conflict of interests

None.

Ethical considerations

All rules and procedures for working on animals (i.e., getting to know, training, anesthesia, and animal killing) were according to AAALAC (Association for Assessment and Accreditation Laboratory Animal Care International) and under the Code of Ethics Number 2398727IRSKU in Shahrekord University.

\section{Acknowledgments}

The authors gratefully acknowledge the Research Deputy of Shahrekord University. We also thank K. Ghatreh for all his laboratory measurements and providing us with some information received from the research project (under the code of 95/05) that was approved on 3.15.2016 in Shahrekord University.

\section{References}

1. Bacchi E, Moghetti P. Exercise for hepatic fat accumulation in type 2 diabetic subjects. Int J Endocrinol. 2013;2013:309191. doi: 10.1155/2013/309191.

2. Goodpaster BH, He J, Watkins S, Kelley DE. Skeletal muscle lipid content and insulin resistance: evidence for a paradox in endurance-trained athletes. J Clin Endocrinol Metab. 2001;86(12):5755-61. doi: 10.1210/jcem.86.12.8075.

3. Samuel VT, Petersen KF, Shulman Gl. Lipid-induced insulin resistance: unravelling the mechanism. Lancet. 2010;375(9733):2267-77. doi: 10.1016/s01406736(10)60408-4 .

4. Shaw CS, Shepherd SO, Wagenmakers AJ, Hansen D, Dendale P, van Loon LJ. Prolonged exercise training increases intramuscular lipid content and perilipin 2 expression in type I muscle fibers of patients with type 2 diabetes. Am J Physiol Endocrinol Metab. 2012;303(9):E1158-65. doi: 10.1152/ ajpendo.00272.2012.

5. Brasaemle DL. Thematic review series: adipocyte biology. The perilipin family of structural lipid droplet proteins: stabilization of lipid droplets and control of lipolysis. J Lipid Res. 2007;48(12):2547-59. doi: 10.1194/jlr.R700014-JLR200.

6. Bosma M. Lipid droplet dynamics in skeletal muscle. Exp Cell Res. 2016;340(2):180-6. doi: 10.1016/j.yexcr.2015.10.023.

7. Pourteymour S, Lee S, Langleite TM, Eckardt K, Hjorth M, Bindesboll C, et al. Perilipin 4 in human skeletal muscle: localization and effect of physical activity. Physiol Rep. 2015;3(8). doi: 10.14814/phy2.12481.

8. Shepherd SO, Cocks M, Tipton KD, Ranasinghe AM, Barker TA, Burniston JG, et al. Sprint interval and traditional endurance training increase net intramuscular triglyceride breakdown and expression of perilipin 2 and 5. J Physiol. 2013;591(3):657-75. doi: 10.1113/jphysiol.2012.240952.

9. Ramos SV, Turnbull PC, MacPherson RE, LeBlanc PJ, Ward WE, Peters SJ. Changes in mitochondrial perilipin 3 and perilipin 5 protein content in rat skeletal muscle following endurance training and acute stimulated contraction. Exp Physiol. 2015;100(4):450-62. doi: 10.1113/expphysiol.2014.084434.

10. Dubé JJ, Amati F, Stefanovic-Racic M, Toledo FG, Sauers 
$\mathrm{SE}$, Goodpaster BH. Exercise-induced alterations in intramyocellular lipids and insulin resistance: the athlete's paradox revisited. Am J Physiol Endocrinol Metab. 2008;294(5):E882-8. doi: 10.1152/ajpendo.00769.2007.

11. Pruchnic R, Katsiaras A, He J, Kelley DE, Winters C, Goodpaster BH. Exercise training increases intramyocellular lipid and oxidative capacity in older adults. Am J Physiol Endocrinol Metab. 2004;287(5):E857-62. doi: 10.1152/ ajpendo.00459.2003.

12. Scribbans TD, Edgett BA, Vorobej K, Mitchell AS, Joanisse SD, Matusiak JB, et al. Fibre-specific responses to endurance and low volume high intensity interval training: striking similarities in acute and chronic adaptation. PLoS One. 2014;9(6):e98119. doi: 10.1371/journal.pone.0098119.

13. van Aggel-Leijssen DP, Saris WH, Wagenmakers AJ, Senden JM, van Baak MA. Effect of exercise training at different intensities on fat metabolism of obese men. J Appl Physiol (1985). 2002;92(3):1300-9. doi: 10.1152/japplphysiol.00030.2001.

14. Ramos S. Changes in mitochondrial PLIN3 and PLIN5 protein content in rat skeletal muscle following acute contraction and endurance training. Ontario, Canada: Brock University; 2014.

15. Carroll KS, Hanna J, Simon I, Krise J, Barbero P, Pfeffer SR. Role of Rab9 GTPase in facilitating receptor recruitment by TIP47. Science. 2001;292(5520):1373-6. doi: 10.1126/ science.1056791.

16. Dalen KT, Dahl T, Holter E, Arntsen B, Londos C, Sztalryd C, et al. LSDP5 is a PAT protein specifically expressed in fatty acid oxidizing tissues. Biochim Biophys Acta. 2007;1771(2):21027. doi: 10.1016/j.bbalip.2006.11.011.

17. Yamaguchi T, Matsushita S, Motojima K, Hirose F, Osumi T. MLDP, a novel PAT family protein localized to lipid droplets and enriched in the heart, is regulated by peroxisome proliferatoractivated receptor alpha. J Biol Chem. 2006;281(20):1423240. doi: 10.1074/jbc.M601682200.

18. Wang H, Bell M, Sreenivasan U, Hu H, Liu J, Dalen K, et al. Unique regulation of adipose triglyceride lipase (ATGL) by perilipin 5, a lipid droplet-associated protein. J Biol Chem. 2011;286(18):15707-15. doi: 10.1074/jbc.M110.207779.

19. de Almeida AR, Monte-Alegre S, Zanini MB, Souza AL, Etchebehere M, Gontijo JA. Association between prehypertension, metabolic and inflammatory markers, decreased adiponectin and enhanced insulinemia in obese subjects. Nutr Metab (Lond). 2014;11:25. doi: 10.1186/17437075-11-25.

20. Molanouri Shamsi M, Mahdavi M, Gharakhanlou R, Hassan MZ. Effect of Intensive Resistance Exercise Training on Protein Expression of IL-6, IL-1 $\beta$ and TNF- $\alpha$ myokines in fast twitch skeletal muscle of diabetic rats. Feyz Journal of Kashan University of Medical Sciences. 2014;6(11):69-77.

21. Sishi B, Loos B, Ellis B, Smith W, du Toit EF, Engelbrecht AM. Diet-induced obesity alters signalling pathways and induces atrophy and apoptosis in skeletal muscle in a prediabetic rat model. Exp Physiol. 2011;96(2):179-93. doi: 10.1113/ expphysiol.2010.054189.

22. Kuramoto K, Sakai F, Yoshinori N, Nakamura TY, Wakabayashi S, Kojidani T, et al. Deficiency of a lipid droplet protein, perilipin 5, suppresses myocardial lipid accumulation, thereby preventing type 1 diabetes-induced heart malfunction. Mol Cell Biol. 2014;34(14):2721-31. doi: 10.1128/mcb.00133-14.

23. MacPherson RE, Herbst EA, Reynolds EJ, Vandenboom R, Roy BD, Peters SJ. Subcellular localization of skeletal muscle lipid droplets and PLIN family proteins OXPAT and ADRP at rest and following contraction in rat soleus muscle. Am J Physiol Regul Integr Comp Physiol. 2012;302(1):R29-36. doi: 10.1152/ajpregu.00163.2011.

24. Minnaard R, Schrauwen P, Schaart G, Jorgensen JA, Lenaers E, Mensink $M$, et al. Adipocyte differentiation-related protein and OXPAT in rat and human skeletal muscle: involvement in lipid accumulation and type 2 diabetes mellitus. J Clin Endocrinol Metab. 2009;94(10):4077-85. doi: 10.1210/jc.2009-0352.

25. Ramos SV, MacPherson RE, Turnbull PC, Bott KN, LeBlanc P, Ward WE, et al. Higher PLIN5 but not PLIN3 content in isolated skeletal muscle mitochondria following acute in vivo contraction in rat hindlimb. Physiol Rep. 2014;2(10). doi: 10.14814/phy2.12154.

26. Mason RR, Meex RC, Russell AP, Canny BJ, Watt MJ. Cellular localization and associations of the major lipolytic proteins in human skeletal muscle at rest and during exercise. PLoS One. 2014;9(7):e103062. doi: 10.1371/journal.pone.0103062.

27. Mason RR, Watt MJ. Unraveling the roles of PLIN5: linking cell biology to physiology. Trends Endocrinol Metab. 2015;26(3):144-52. doi: 10.1016/j.tem.2015.01.005.

28. Bosma M, Hesselink MK, Sparks LM, Timmers S, Ferraz MJ, Mattijssen $\mathrm{F}$, et al. Perilipin 2 improves insulin sensitivity in skeletal muscle despite elevated intramuscular lipid levels. Diabetes. 2012;61(11):2679-90. doi: 10.2337/db11-1402.

29. Gjelstad IM, Haugen F, Gulseth HL, Norheim F, Jans A, Bakke SS, et al. Expression of perilipins in human skeletal muscle in vitro and in vivo in relation to diet, exercise and energy balance. Arch Physiol Biochem. 2012;118(1):22-30. doi: 10.3109/13813455.2011.630009.

30. Dan M, Chantler JK. A novel pancreatropic coxsackievirus vector expressing glucagon-like peptide 1 reduces hyperglycemia in streptozotocin-treated mice. J Virol. 2011;85(23):12759-68. doi: 10.1128/jvi.00661-11.

31. Bergman BC, Perreault L, Hunerdosse DM, Koehler MC, Samek AM, Eckel RH. Increased intramuscular lipid synthesis and low saturation relate to insulin sensitivity in endurancetrained athletes. J Appl Physiol (1985). 2010;108(5):1134-41. doi: 10.1152/japplphysiol.00684.2009.

32. Banitalebi E, Razavi T, Norian M, Bagheri L. The effect of combined aerobic exercise training and green tea extract on serum TNF-a and IL-6 levels in obese women with type 2 diabetes. Daneshvar Medicine. 2016;23(123):11-20. [Persian]. 